\title{
Near-field interrogation of SAW resonators on rotating machinery
}

\author{
J.-M. Boccard ${ }^{1}$, P. Katus ${ }^{1}$, R. Renevier ${ }^{1}$, L. M. Reindl ${ }^{1}$, and J.-M. Friedt ${ }^{2}$ \\ ${ }^{1}$ Institute of Microsystems Engineering IMTEK Laboratory for Electrical Instrumentation, \\ University of Freiburg, Freiburg, Germany \\ ${ }^{2}$ SENSeOR, hosted by the Time \& Frequency department of FEMTO-ST, Besançon, France
}

Correspondence to: J.-M. Boccard (jean-michel.boccard@imtek.uni-freiburg.de)

Received: 19 June 2013 - Revised: 20 August 2013 - Accepted: 4 September 2013 - Published: 17 September 2013

\begin{abstract}
Surface acoustic wave (SAW) resonators electrically behave like LCR circuits, their frequency can be influenced by temperature, pressure and torque. When they are used for passive wireless sensing on rotating machinery, they can also be influenced by the angular variations of the coupling between the coupler elements and the receiving coupler element impedance. This parasitic frequency shift is known as the "pulling effect". In this paper, we present a capacitive coupler based on open coplanar strip lines for physical measurements on a small diameter rotating shaft. This approach allows a single $434 \mathrm{MHz}$ resonator angular frequency pulling lower than $200 \mathrm{~Hz}(0.46 \mathrm{ppm})$ and $100 \mathrm{~Hz}(0.23 \mathrm{ppm})$ in a differential configuration. This is more than 10 times lower compared to frequency pulling obtained using couplers based on circular and electrically shorted transmission lines. RADAR-based interrogation, finite element method (FEM) simulation, coupler parameters and frequency pulling measurements results are presented to demonstrate the performances of the complete system.
\end{abstract}

\section{Introduction}

Surface acoustic wave (SAW) devices have been largely used for filtering radio frequency (RF) signals such as in mobile phone applications and analog signal processing as discussed in Campbell (1989), Royer and Dieulesaint (2010). For the last $20 \mathrm{yr}$, SAW transducers have also been used as wireless sensors in harsh environments such as for temperature measurements as reported in Reindl and Shrena (2004), Friedt et al. (2011), Fachberger et al. (2006). They have also been used for torque (Beckley et al., 2002; Kalinin and Brown, 2010), strain (Pohl et al., 1999; Friedt et al., 2012), pressure (Reindl et al., 1998; Buff et al., 1997), chemical (Dong et al., 2001; Wang et al., 2009) and moisture level measurements (Kawalec and Pasternak, 2008). Due to the deeper penetration depth of electromagnetic waves at low operating frequency, the interrogation of SAW sensors is mainly focused on the European industrial, scientific and medical (ISM) band centered in $434 \mathrm{MHz}$ whose narrow bandwidth is only compatible with a resonator configuration. It is help- ful for measurements in dielectric media such, as tires as discussed in Dixon et al. (2006).

Beyond the physical quantity effect on the acoustic velocity and hence resonance frequency change due to the phenomena under investigation, parasitic impedances will induce an impedance change of the probed circuit. It includes the resonator and the transmission line made of the transceiver and coupler receiver element as well as the nearfield space in-between. Since a basic model of the sensor is a series inductors and capacitors, adding a transmission line whose characteristics varies with angular position will vary the global circuit impedance and hence the resonance frequency detected by the reader. This unwanted frequency change is called the "pulling effect" since it will always yield frequency decrease with respect to the resonator resonance frequency. Although classically used to tune oscillator frequency in closed loop circuits, it is an unwanted parasitic effect in wireless sensor interrogation.

In this paper, a capacitive coupler based on open coplanar strip lines for the interrogation of $434 \mathrm{MHz}$ SAW resonators 
mounted on a $3 \mathrm{~cm}$ diameter shaft is presented. Due to the detuning of the coupler elements to the sensor resonance frequency (i.e., low coupling), low angular variations of coupler parameters in the sensor frequency range are possible. Therefore, the coupling system allows for a low parasitic effect on the sensor. The paper is organized as follows: In Sect. 2, the state-of-the-art on SAW resonator sensors is given, followed by the review of near-field couplers. In Sect. 3, the wireless interrogation principle based on a pulsed RADAR strategy is summarized. Further, the use of ceramic antennas as receiving elements followed by the description of a capacitive coupler based on open coplanar strip lines are discussed. Section 4 presents finite element method (FEM) simulation of the coupler fields distributions using high frequency structural simulator (HFSS, Ansys). Further, the coupler electrical parameters measurements are given and the sensitivity to the mechanical setup and the surrounding environment are assessed. The results of calculated and measured angular frequency pulling and offsets at different gaps between the coupler elements are given, followed by moisture level measurements.

\section{State-of-the-art}

\subsection{SAW resonators sensors}

SAW sensors are based on monitoring the effect of an external physical quantity under investigation on a mechanical wave propagation velocity. Interdigitated transducers (IDTs) patterned on a piezoelectric substrate convert through the inverse piezoelectric effect an electric signal to a mechanical wave. The propagation in the crystal has been described in White and Voltmer (1965). The mechanical wave is then converted back on the same or another set of IDTs to an electric signal by the direct piezoelectric effect. Single crystal piezoelectric substrates are favored for sensing applications since detailed modeling is possible thanks to the availability of sets of constants (Ballandras et al., 2009).

For the resonator configuration, in which the transduction IDT is surrounded by Bragg mirrors, the acoustic energy is confined in a cavity. It provides the means for a narrowband and compact device (Beckley et al., 2002) and most significantly exhibiting low losses. Hence, low electromechanical coupling coefficients substrates such as quartz are frequently used. This material exhibits several cuts of low temperature sensitivity. The characteristic measured quantity is the resonance frequency and exponential decay time constant which is equal to $Q / \pi$ periods. Typical quality factors for Rayleighmode SAW transducers packaged at atmospheric pressure are in the range of 10000 for $434 \mathrm{MHz}$ devices. Despite low temperature sensitivity, a dual resonator approach in which two acoustic waves propagate along different directions on a common substrate is mandatory for reducing the temperature influence on a wide operating-temperature range as described in Buff et al. (1997).

\subsection{Near-field couplers}

The integration of SAW devices working at $434 \mathrm{MHz}$ on a few centimeter diameter rotating shaft in a metallic environment is compromised by the size and the sensitivity of the antennas, especially for far-field communications when a $360^{\circ}$ angular coverage is required. In fact, the corresponding wavelength at $434 \mathrm{MHz}$ is about $70 \mathrm{~cm}$. Hence, a circular near-field coupler is more suitable, where the energy is transferred by inductive or capacitive coupling in the reactive region, as described in Balanis (1997), Lecklider (2005).

Rotary RF couplers (Lonsdale and Lonsdale, 2001; Sandacci and Gilkes, 2006; Beckley and Kalinin, 2006) and circular microstrip couplers (Beckley et al., 2002) have been used for the wireless interrogation of SAW resonators (mainly strain sensors) on small diameter shafts and several couplers were patented. The common designs remain on two circular and electrically shorted transmission lines. These devices are tuned to the frequency and impedance of the resonator to provide a sufficient coupling. On the other hand, the resonator sensor frequency pulling is due to the high coupling, receiver coupler element resonance frequency, quality factor and impedance variations as discussed in Tourette (2010). These parameters are a function of angular position in rotating machinery application. Typical single resonator frequency pulling is over $2 \mathrm{kHz}(4.6 \mathrm{ppm}$ at $434 \mathrm{MHz})$ if no de-coupling resistor or capacitor is added (Kalinin, 2008).

\section{Designs}

The diagram of the SAW resonator interrogation principle based on RADAR strategy is shown in Fig. 1. In this section, the interrogation electronics are described, followed by two near-field coupler concepts.

\subsection{Interrogation electronics}

In this work, frequency domain measurements by searching the maximal insertion were used loss, as described in Friedt et al. (2010). Pulses long enough for their spectral width to be narrower than the resonator bandwidth transfer all incoming energy to the resonator at a known accurate emitted pulse frequency. In this case, the recorded quantity is the returned power. Since the SAW resonator behavior is linear, there is no need to recover frequency information. Therefore a wideband power amplifier and detector followed by an analog to digital converter is enough on the reception stage.

An important hardware characteristic defining the interrogation range is the isolation between the emission and reception stages. Here, three duplexers are used to connect the source to the antenna during the loading stage, and the antenna to the receiver during the listening stage. A $80 \mathrm{~dB}$ isolation has been measured experimentally. With a quality factor $Q$ of 10000 , the time constant of a $434 \mathrm{MHz}$ resonator loading and unloading is $\tau=Q$ periods, equal to about $7 \mu$ s. 


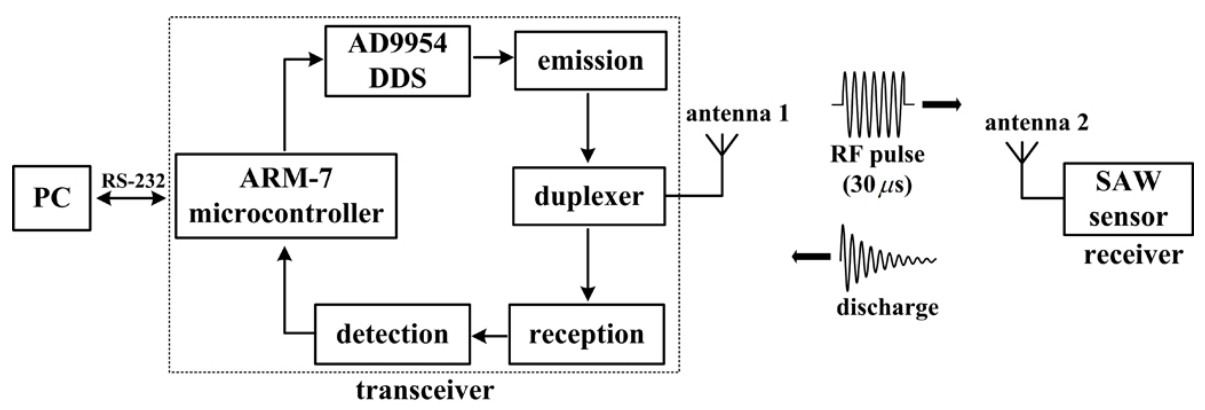

Figure 1. Diagram of the resonator interrogation principle based on the RADAR strategy.

The emitted pulse is tuned to be narrower than the width at half height of the resonator by selecting an emission time of $5 \tau$. Furthermore, each successive measurement is independent from the previous by a delay of $5 \tau$. Thus, the resonator is fully unloaded before starting the next interrogation step. The interrogation routine repeats this measurement 128 times and sweeps along the whole ISM band. A frequency step of one third of the width at half height of the resonator is optimal for the following digital signal processing. Since a frequency sweep using frequency steps equal to the aimed frequency resolution - sub-kHz - would require excessive sweep duration, the ISM band is swept using coarse frequency steps.

The measured insertion loss is fitted by a second order polynomial, whose maximum position provides an accurate estimate of the resonance frequency within $7 \mathrm{~ms}$ sweep time. The resolution is almost equal to the signal-to-noise ratio on the returned power measurements. However, a bias has been observed due to the application of the parabola fit to the actual resonator modeled by a Butterworth-van Dyke model. This bias is eliminated by using a 3-point strategy in which the interrogation frequency comb is no longer fixed but tracks the last resonance frequency estimate $f_{0}$. With such procedure, the measurement focuses on the last resonance frequency computation using the parabola fit and the next measurement is performed at $f_{0}$ and $f_{0} \pm \Delta f$. Thanks to this strategy, the bias is kept constant throughout the measurement and the resonator must only be visible from the interrogation unit for a duration of $420 \mu \mathrm{s}$.

Under such conditions, in our setup the asynchronous (RS232) serial communication limits the measurement bandwidth to 10 values per second. Nevertheless it provides the highest resolution with respect to a digital to analog voltage output with an update rate of $2400 \mathrm{~Hz}$. Moreover, a 1 to $32 \mathrm{~dB}$ programmable attenuator (located before the power 180 duplexer on the emission stage) is used to increase the dynamic range in order to avoid saturation of the received signal. However, the automatic gain control of our hardware cannot compensate fast signal variations. The short-time dynamic range is $4 \mathrm{~dB}$. Therefore, in order to guarantee the full interrogation coverage in rotating machinery applications using this hardware, the coupler should be designed in order

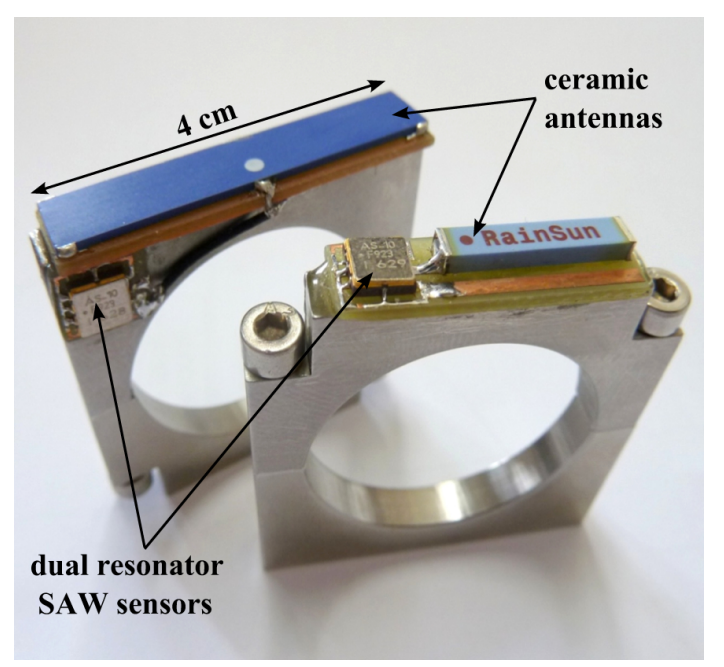

Figure 2. Multilayer ceramic antennas connected to double resonance SAW temperature sensors on steel clamps.

that the angular variation of transmission amplitude $\left(S_{21}\right)$ is lower than $\pm 2 \mathrm{~dB}$.

\subsection{Ceramic antennas as receiving elements}

Ceramic antennas exhibit reduced dimensions with respect to the wavelength of the electromagnetic wave in free space thanks to high permittivity materials as used in Ohnimus et al. (2010). They are made by low temperature co-fired ceramic (LTCC) technology or chip capacitor process as discussed in Zhang (2011) and contain interconnected metallic meanders or coil configurations. Therefore, they can be used to reduce the size of the receiver element compared to simple antennas.

Figure 2 shows two types of multilayer ceramic antennas (Rainsun AN1603-433 and Yageo 4313121200431B) connected to double resonator temperature SAW sensors and fixed on steel clamps. The substrates are made of $1 \mathrm{~mm}$ thick FR-4 epoxy. A strip line connected to the ground is placed in parallel to the antenna. Their radiation is poor in this configuration due to the presence of the clamp, but it is enough 


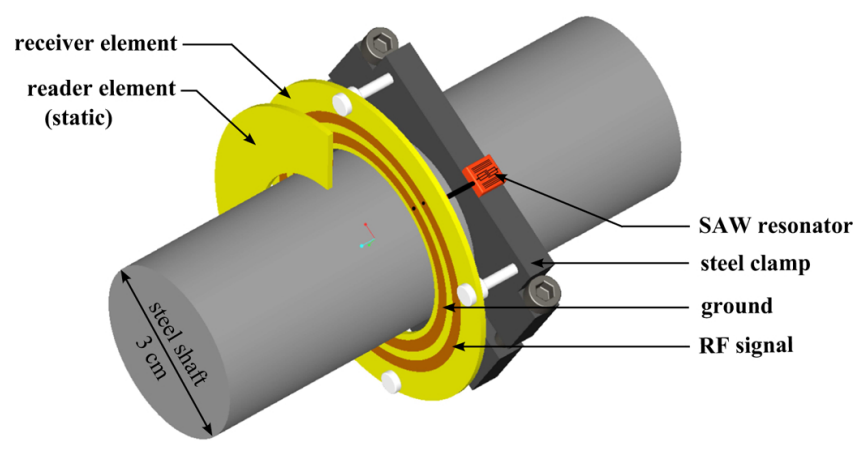

Figure 3. 3-D model of a capacitive coupler based on coplanar strip lines, placed around a $3 \mathrm{~cm}$ diameter shaft and connected to a SAW resonator.

for near-field transmissions in the centimeter range. As it has been reported in Boccard and Reindl (2012), a printed loop connected to the reader unit can be placed in parallel to the clamp system around the shaft. However, in this configuration the angular variation of transmission amplitude is higher than $10 \mathrm{~dB}$, which is due to the non-uniform current distribution around the loop. Therefore, the full interrogation coverage of a sensor around the shaft using our setup is compromised. Moreover, the high quality factor of ceramic antennas increase the sensor angular frequency pulling (Tourette, 2010).

\subsection{Capacitive coupler based on coplanar strip lines}

\subsubsection{Design}

Figure 3 shows the model of a circular capacitive coupler comprising one static and one rotating element. Both elements are based on open coplanar strip lines as described in Knorr and Kuchler (1975), DiPaolo (2000), Suh and Chang (2002). These arrangements are frequently used for filtering and signal transmission. The rotating part is mounted on a $3 \mathrm{~cm}$ diameter shaft using nonmetallic fixations (such as polyamide or ceramic) on a steel clamp and is connected to one or several resonators working in the $434 \mathrm{MHz}$ range. The static part is connected to the reader unit and is a cut $\left(120^{\circ}\right)$ from the first element but could also be a complete circular element. In this balanced configuration, the coupling is mainly due to the electrical field portion which is orthogonal to the circular transmission line.

Figure 4 shows the manufactured coupler elements. The conductors are $2 \mathrm{~mm}$ wide, separated by $1.5 \mathrm{~mm}$ and printed on a single layer of $1 \mathrm{~mm}$ thick FR4 epoxy. The receiver outer ring has a length of $14 \mathrm{~cm}$, which corresponds to an electrical length of $7 \mathrm{~cm}$ due to the symmetry. This is smaller than the quarter wavelength at the sensor resonance frequency. The receiver element is resonating at $840 \mathrm{MHz}$ and the reader element at $1.6 \mathrm{GHz}$. Both coupler elements are detuned from $434 \mathrm{MHz}$ in order to achieve low parasitic frequency pulling

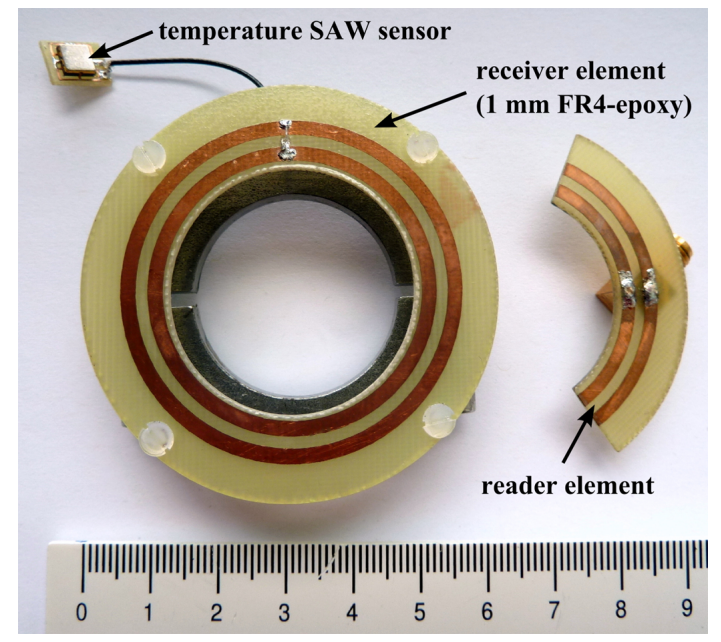

Figure 4. Picture of the manufactured coupler elements on $1 \mathrm{~mm}$ thick FR-4 epoxy substrates.

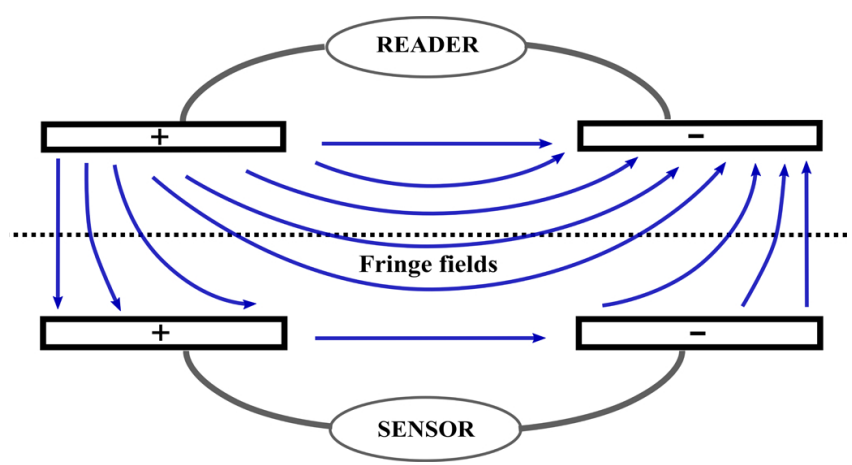

Figure 5. Sketch of electrical field coupling between the coupler elements.

on the connected resonators. The coupler elements operate below resonance to assure minor dependence on rotation angle caused by the small electrical field variation along the lines. The capacitive coupling between the disks is sketched in Fig. 5. The capacitive coupling current is induced by the radial part of the electrical field. The inductive currents are neglected since they cancel each other in large part. A double resonator temperature SAW sensor is connected to the receiver coupler element using a Hirose WFL-2LP-04N2-A $50 \Omega$ coaxial cable which has a length of $3 \mathrm{~cm}$.

\subsubsection{Equivalent circuit model}

The linearized equivalent circuit model of the previously presented capacitive coupler connected to a SAW resonator and in the $434 \mathrm{MHz}$ range is shown in Fig. 6. This schematic is used to analyze the angular parasitic effect on a connected resonator due to the coupler electrical parameters variations in a specific working frequency range. The coupler schematic is represented by the three capacitors $C_{1}, C_{2}$ 


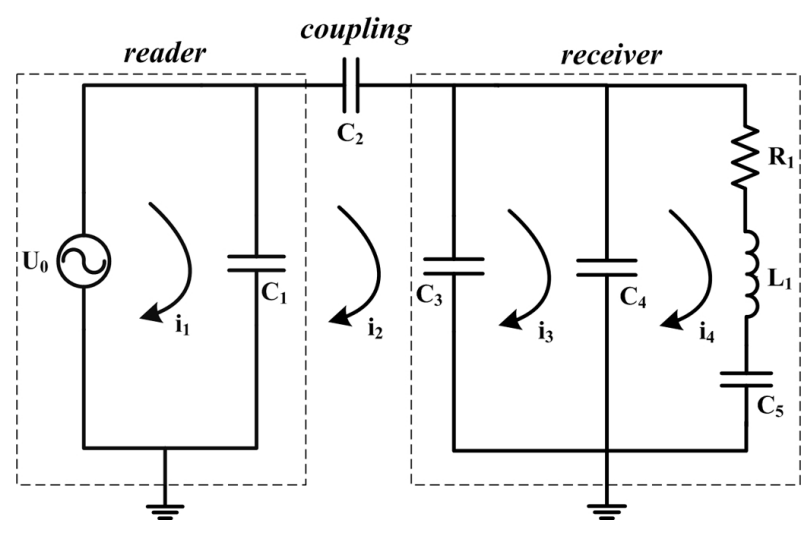

Figure 6. Simplified linearized equivalent circuit model of the capacitive coupler connected to a SAW resonator.

and $C_{3}$, respectively corresponding to the reader element, the coupling and the receiver element. The losses are not modeled since their influence is negligible. The resonator is modeled according to Butterworth-van Dyke with the static capacitor $C_{4}(4 \mathrm{pF})$. The circuit holding the antenna connected in parallel to the sensor adds another $1.8 \mathrm{pF}$, yielding a total static capacitance of $5.8 \mathrm{pF}$. The other model components are the motional capacitance $C_{5}(0.6 \mathrm{fF})$, the motional inductor $L_{1}(224 \mu \mathrm{H})$ and the dissipation resistor $R_{1}(38 \Omega)$ which will be neglected in the following equations. The series resonance is approximately $1 /\left(2 \pi \sqrt{L_{1} C_{5}}\right)$. Referring to the parallel resonance, the coupler capacitors values $C_{2}$ and $C_{3}$ have to remain the most constant as possible in order to reduce the sensor parasitic frequency pulling $\left(C_{1}\right.$ is neglected due to the small value of coupling capacitor $C_{2}$ ). Low parameter variations are possible if the couplers elements are not tuned to the sensor frequency, providing a low coupling as discussed in Tourette (2010).

$U_{1}=\frac{1}{s C_{1}} i_{1}-\frac{1}{s C_{1}} i_{2}$

$U_{2}=-\frac{1}{s C_{1}} i_{1}+\left(\frac{1}{s C_{1}}+\frac{1}{s C_{2}}+\frac{1}{s C_{3}}\right) i_{2}-\frac{1}{s C_{3}} i_{3}$

$U_{3}=-\frac{1}{s C_{3}} i_{2}+\left(\frac{1}{s C_{3}}+\frac{1}{s C_{4}}\right) i_{3}-\frac{1}{s C_{4}} i_{4}$

$U_{4}=-\frac{1}{s C_{4}} i_{3}+\left(\frac{1}{s C_{4}}+\frac{1}{s C_{5}}+s L_{1}\right) i_{4}$

Equations (1) to (4) give the voltage and the current expressions from the equivalent circuit model (with $s=j \omega$ ). By solving these equations, the admittance seen from the reader side is calculated. It is composed of a static capacitance and a resonant term. The resonant term $P$ is given in Eq. (5). By setting this equation to zero and solving for complex frequency, it gives the solution shown in Eq. (6). This resonance frequency will be used to calculate the sensor frequency pulling depending on the coupler variables $C_{2}$ and $C_{3}$.

$$
\begin{aligned}
P & =C_{2}+C_{3}+C_{4}+C_{5}+C_{2} C_{5} L_{1} s^{2} \\
& +C_{3} C_{5} L_{1} s^{2}+C_{4} C_{5} L_{1} s^{2} \\
f_{\text {res }} & =\frac{\sqrt{C_{2}+C_{3}+C_{4}+C_{5}}}{2 \pi \sqrt{\left(C_{2}+C_{3}+C_{4}\right) \times C_{5} \times L_{1}}}
\end{aligned}
$$

Equations (7) and (8) give the expressions of $C_{2}$ and $C_{3}$ depending on $\operatorname{Im}\left(Y_{21}\right)$ (transmission susceptance) and $\operatorname{Im}\left(Y_{22}\right)$ (receiver element susceptance) of the coupler without connected resonator. These equations are based on the pi-network formulas.

$C_{2}=\frac{\operatorname{Im}\left(Y_{21}\right)}{2 \pi f}$

$C_{3}=\frac{\operatorname{Im}\left(Y_{22}\right)-\operatorname{Im}\left(Y_{21}\right)}{2 \pi f}$

\section{Results and discussion}

\subsection{FEM simulation results}

As previously described, the transmission between the coupler elements has to remain constant at each rotation angle in order to avoid the resonator sensor frequency pulling. Therefore, the generated electrical field $(E)$ by the receiver element must be uniformly distributed so that the angular capacitive coupling variation is low. FEM simulations have been done using HFSS. In order to avoid disturbances due to the presence of the metallic clamp (i.e., capacitive effect, Eddy currents), the gap between the clamp and the receiver element has been set to $1 \mathrm{~cm}$. The cables and connectors that are used during the experiments have not been included. Figure 7 shows the generated electrical field by the receiver element at $434 \mathrm{MHz}$ and at $3 \mathrm{~mm}$ from its surface. The open coplanar strip lines allow a circular field distribution along it without drastic drops. The maximum voltage occurs at the opposite of the feeding point. Figure 8 shows the generated magnetic field $(H)$ in the same configuration. Due to the boundary condition, this field drops at the opposite of the feeding point where there is zero current and is partly by $180^{\circ}$ phase shifted to the $\mathrm{E}$ field. Moreover, the radiation efficiency $\eta \approx 3 \%$ is low due to the fact that its resonance is far from $434 \mathrm{MHz}$ and due to impedance mismatch.

\subsection{Measurement results}

\subsubsection{Coupler parameters measurements}

The transmission amplitude $S_{21}$ between the coupler elements has been measured at $434 \mathrm{MHz}$ using a network analyzer and by calibrating the used connectors and coaxial 


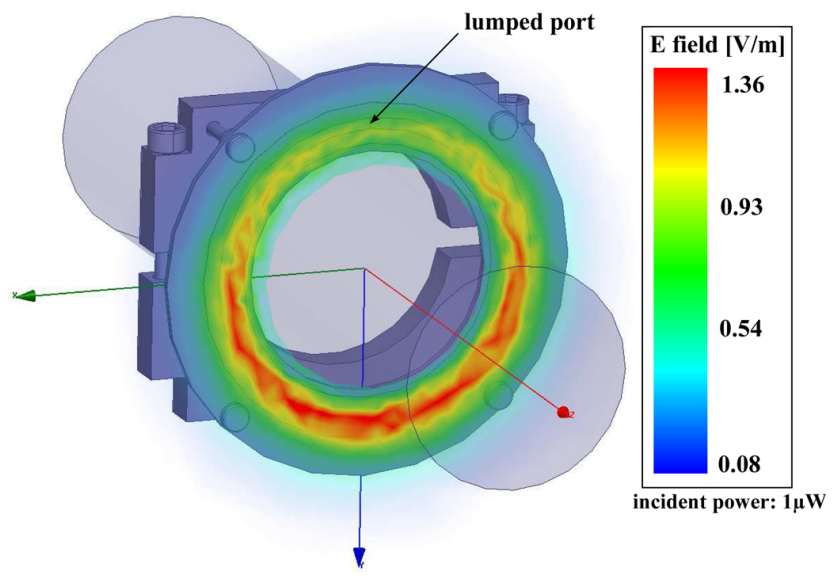

Figure 7. Simulated electrical field at $3 \mathrm{~mm}$ from the receiving capacitive coupler element surface.

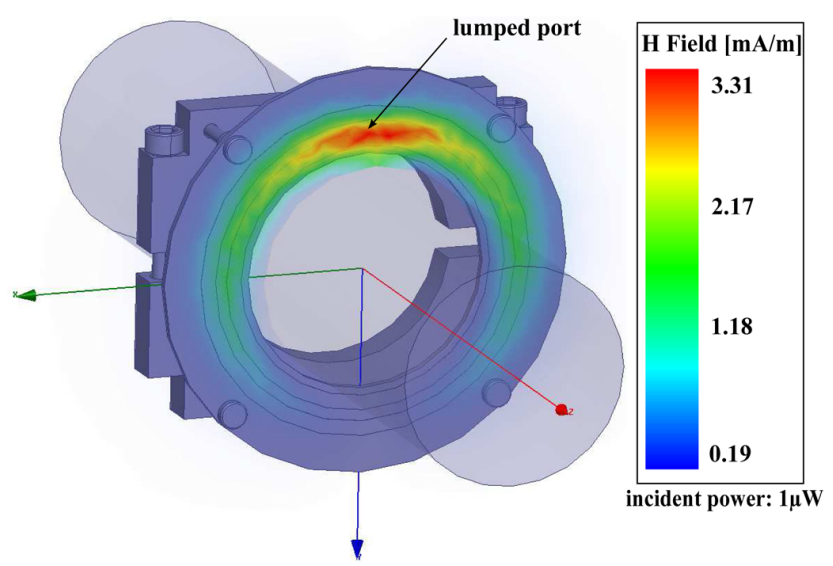

Figure 8. Simulated magnetic field at $3 \mathrm{~mm}$ from the receiving capacitive coupler element surface.

cables to $50 \Omega$. The measurement setup is shown in Fig. 9 . As shown in Fig. 10, the transmission changes by less than $3 \pm 0.6 \mathrm{~dB}$ during a revolution at different gaps between $2 \mathrm{~mm}$ and $10 \mathrm{~mm}$. According to the electrical field distribution shown in Fig. 7, the lowest but acceptable transmission occurs when the two feeding points are in front of each other. This position is defined as zero rotation angle.

Figures 11 and 12 show that the transmission susceptance $\operatorname{Im}\left(Y_{21}\right)$ shifts by $58.22 \%$ during the rotation at $434 \mathrm{MHz}$ and for a gap of $3 \mathrm{~mm}$. The values are negative, proving that the coupling is capacitive in this frequency range. The variations near $800 \mathrm{MHz}$ and between $1.6 \mathrm{GHz}$ and $2 \mathrm{GHz}$ observed in Fig. 11 are respectively due to the receiver and transceiver elements resonances. We assume that the small ripples come from reflections in the RF path due to impedance mismatch. Figures 13 and 14 show that the susceptance of the receiver element $\operatorname{Im}\left(Y_{22}\right)$ is shifting only by $1.55 \%$ and is positive, proving that the characteristic of the coupler receiver element is capacitive in this frequency range. Table 1 contains

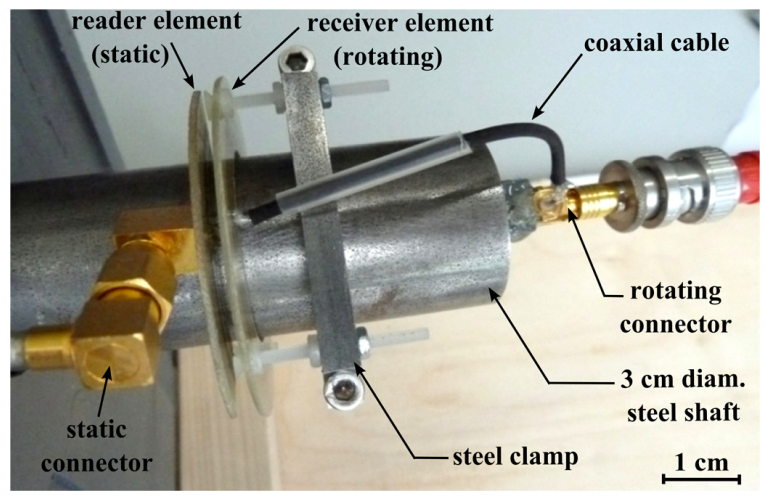

Figure 9. Coupler parameters measurement setup. The coupler elements are connected to the network analyzer using calibrated cables.

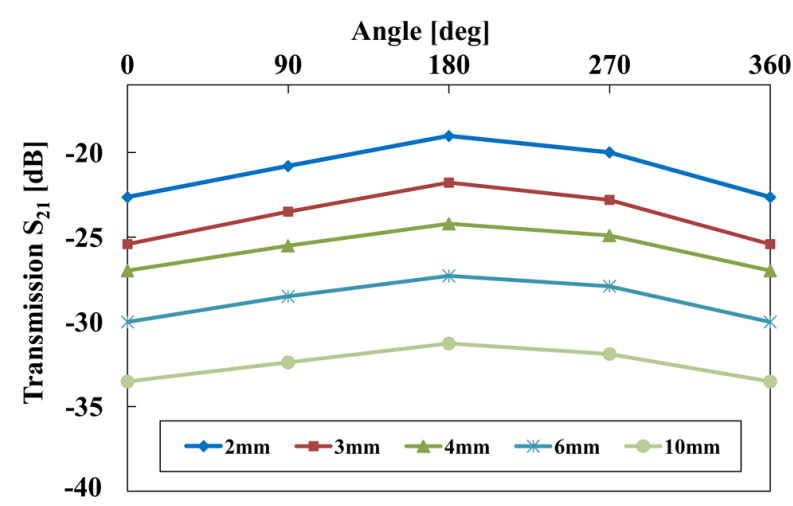

Figure 10. Measured angular transmission amplitude $S_{21}$ variation between the coupler elements at different gaps.

the measured susceptance variations for different gaps at $0^{\circ}$ and $180^{\circ}$ angular positions (where the variations are maximum) at $434 \mathrm{MHz}$. Figure 15 shows the angular variations of the calculated coupler capacitors $C_{2}$ and $C_{3}$. All the capacitors values decrease and reach minimum values at larger gaps due to the decreasing coupling. As expected, the coupling capacitors are lower than the receiver coupler element values. These variations have been used to calculate a connected sensor frequency pulling and offset shown in the following section.

\subsubsection{Resonator frequency pulling measurements}

In this section, the resonator frequency pulling as function of the mechanical setup and the effects due to humidity level are assessed and compared to the calculated values. Figure 16 shows the manufactured measurement bench. A step motor is linked to a $3 \mathrm{~cm}$ diameter steel axis. A dual resonator temperature sensor (TSEAS10 sensor) in which two Rayleigh-mode SAW resonators patterned on a common quartz substrate are connected in parallel to the receiver coupler element. One resonator is orientated at $20^{\circ}$ with respect to the $X$ crystal axis 


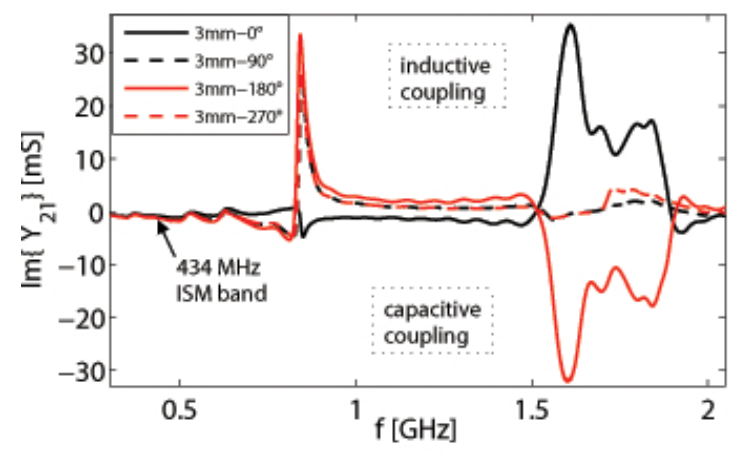

Figure 11. Measured angular variation of transmission susceptance $\operatorname{Im}\left(Y_{21}\right)$ in large frequency scale for a $3 \mathrm{~mm}$ gap.

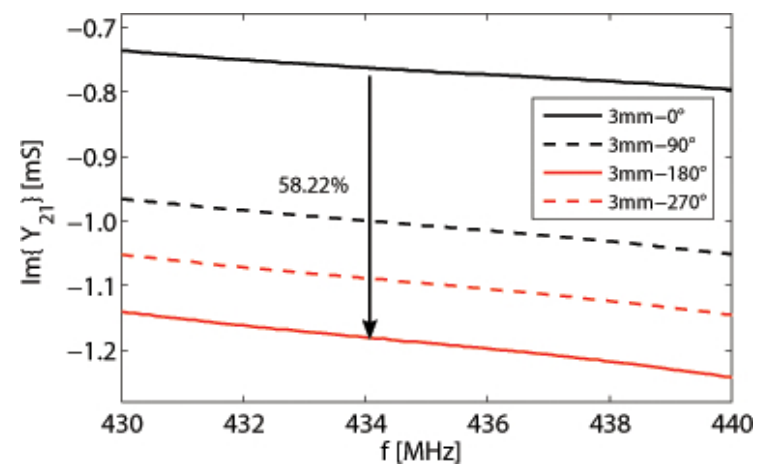

Figure 12. Measured angular of transmission susceptance $\operatorname{Im}\left(Y_{21}\right)$ in extended ISM frequency range for a $3 \mathrm{~mm}$ gap.

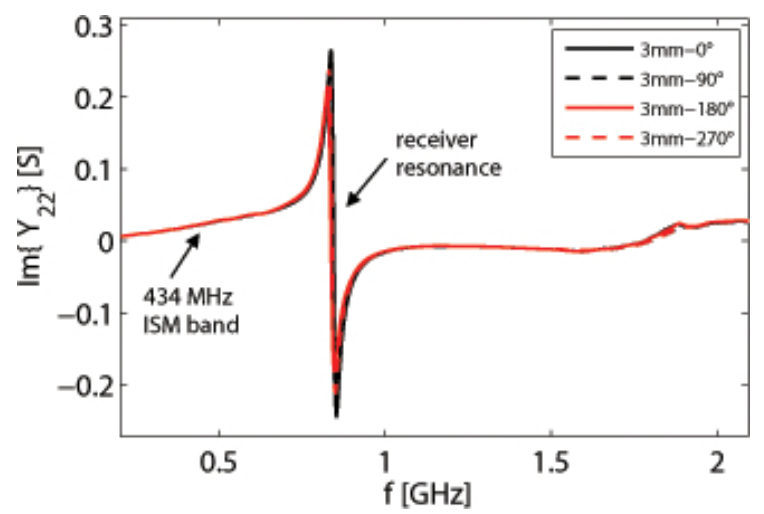

Figure 13. Measured angular of receiver susceptance $\operatorname{Im}\left(Y_{22}\right)$ in large frequency scale for a $3 \mathrm{~mm}$ gap.

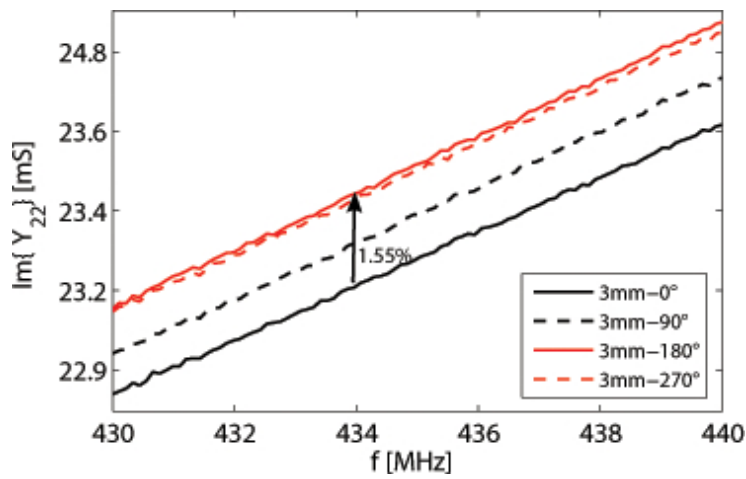

Figure 14. Measured angular variation of receiver susceptance $\operatorname{Im}\left(Y_{22}\right)$ in extended ISM frequency band for a $3 \mathrm{~mm}$ gap.

Table 1. Measured coupler transmission $\operatorname{Im}\left(Y_{21}\right)$ and receiver $\operatorname{Im}\left(Y_{22}\right)$ susceptance variations for different gaps at $434 \mathrm{MHz}$.

\begin{tabular}{ccc}
\hline Gap [mm] & $\operatorname{Im}\left(Y_{21}\right)[\%]$ & $\operatorname{Im}\left(Y_{22}\right)[\%]$ \\
\hline 2 & 54.98 & 2.49 \\
3 & 58.22 & 1.55 \\
4 & 39.67 & 2.08 \\
5 & 47.85 & 0.78 \\
6 & 38.71 & 0.56 \\
7 & 34.90 & 0.43 \\
8 & 41.07 & 0.30 \\
9 & 34.42 & 0.20 \\
10 & 36.05 & 0.65 \\
\hline
\end{tabular}

propagation direction and is therefore temperature dependent ( $f_{1}$ at $433.5 \mathrm{MHz}$ ); the other resonator is used as a reference $\left(f_{2}\right.$ at $\left.434.2 \mathrm{MHz}\right)$ with a turnover temperature close to room temperature. The sensor differential temperature sensitivity is $2.5 \mathrm{kHz} \mathrm{K}^{-1}\left(5.7 \mathrm{ppm} \mathrm{K}^{-1}\right)$ when operating between $-20^{\circ} \mathrm{C}$ and $+160^{\circ} \mathrm{C}$. All the following measurements have been realized in a climatic chamber Weiss WK340, at $25^{\circ} \mathrm{C}$ and at $55 \%$ of humidity. Figure 17 shows the angular frequency pulling results for a $3 \mathrm{~mm}$ gap between the coupler elements. Four revolutions have been realized using the stepper motor (3.6 $6^{\circ}$ steps at $1 \mathrm{~Hz}$ rate). The automatic gain control, controlling the radiofrequency emission power was activated, so that the return power is at mid-scale of the analog to digital converter. Moreover the values are averaged 16 times. Digital post processing using a median filter reduced the resulting standard deviation from $\approx 550$ to $\approx 10 \mathrm{~Hz}$. The observed pulling effect due to the angular rotation on both resonators is below $160 \mathrm{~Hz}(0.37 \mathrm{ppm})$. Figure 18 shows that a differential measurement $\left(f_{2}-f_{1}\right)$ can further reduce the frequency difference pulling effect to below $100 \mathrm{~Hz}(0.23 \mathrm{ppm})$.

Figure 19 shows the calculated (equivalent circuit model used) and the measured frequency offsets (using the reference resonator $f_{2}$ ) at $0^{\circ}$ and $180^{\circ}$ rotation angles and using a reference gap of $2 \mathrm{~mm}$. In this case, the frequency offset 


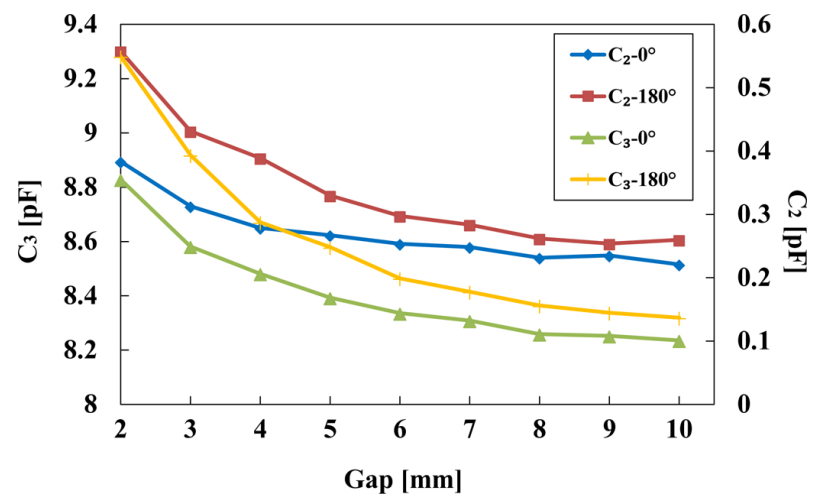

Figure 15. Calculated coupler capacitors $C_{2}$ (transmission) and $C_{3}$ (receiver element) variations at different gaps.

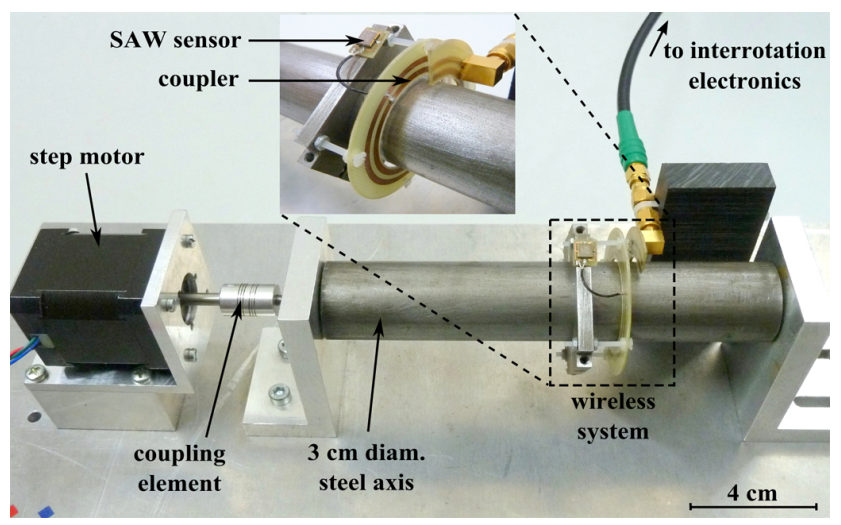

Figure 16. Picture of the bench used for frequency pulling measurements. A step motor is coupled to a $3 \mathrm{~cm}$ diameter steel axis. A dual resonance temperature SAW sensor is fixed on a clamp system and is wirelessly interrogated using the capacitive coupler.

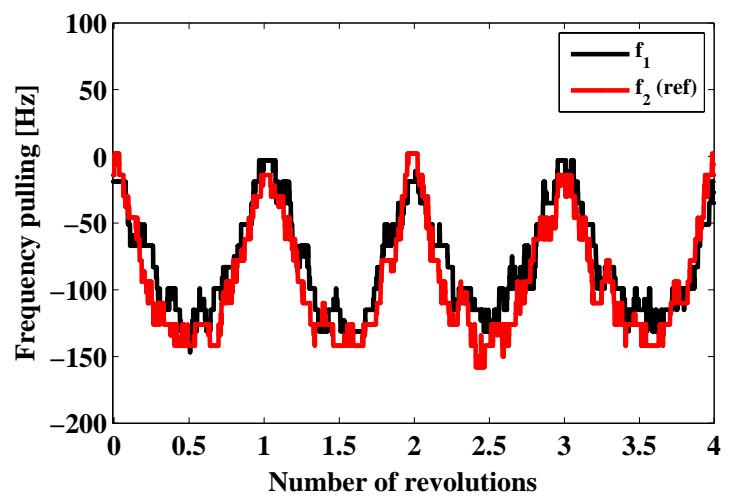

Figure 17. Measured angular frequency pulling obtained from a dual resonator temperature SAW sensor (gap: $3 \mathrm{~mm}, 25^{\circ} \mathrm{C}$ ).

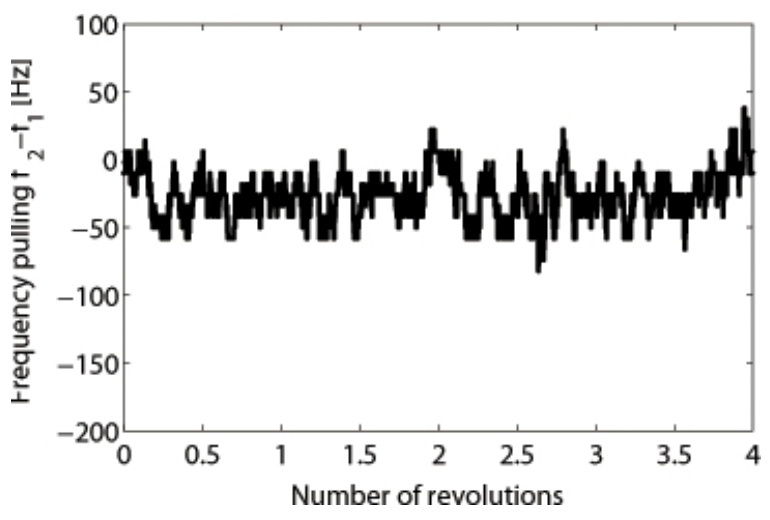

Figure 18. Calculated frequency difference $\left(f_{2}-f_{1}\right)$ from angular frequency pulling measurements (gap: $3 \mathrm{~mm}, 25^{\circ} \mathrm{C}$ ).

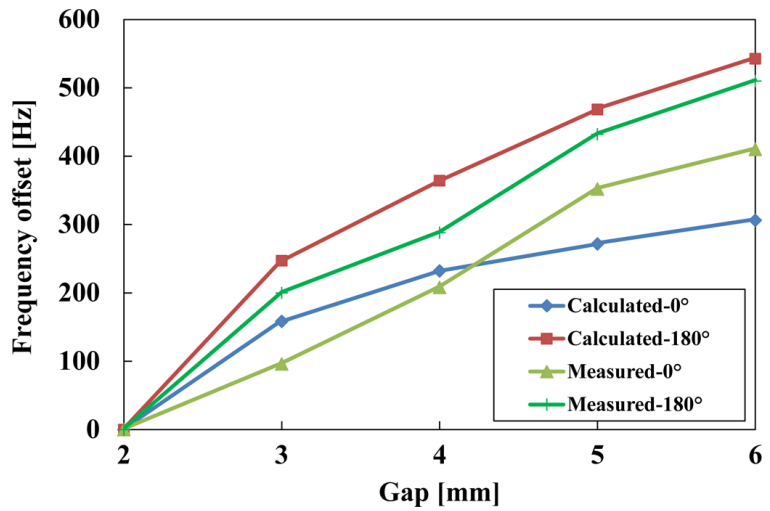

Figure 19. Calculated and measured frequency offsets at different gaps.

is defined by the frequency shift occurring for a specific angular position when the gap between the coupler elements is increased. Figure 20 shows that the expected and measured frequency pulling decreases when the gap is increased, since the coupler capacitors decrease. The difference is about $120 \mathrm{~Hz}$ for a $2 \mathrm{~mm}$ gap and both curves reach similar values for larger gaps. The observed discrepancy between calculation and measurement of Figs. 19 and 20 may come from the cables and connectors and must be further analyzed.

As discussed in El-Sa'ad et al. (1990), FR-4 epoxy material is highly sensitive to the surrounding moisture level, changing the substrate permittivity. In our setup, the coupler receiver element properties may change at different humidity level, disturbing a connected sensor. In order to measure this effect, the humidity has been increased each $30 \mathrm{~min}$ in five steps from 55 to $98 \%$ and with a gap of $3 \mathrm{~mm}$ between the coupler elements. Figure 21 shows a low linear offset below $100 \mathrm{~Hz}(0.23 \mathrm{ppm})$ for the reference resonator $f_{2}$ and at two angular positions. This is in the same order than the measured angular frequency pulling measurements. 


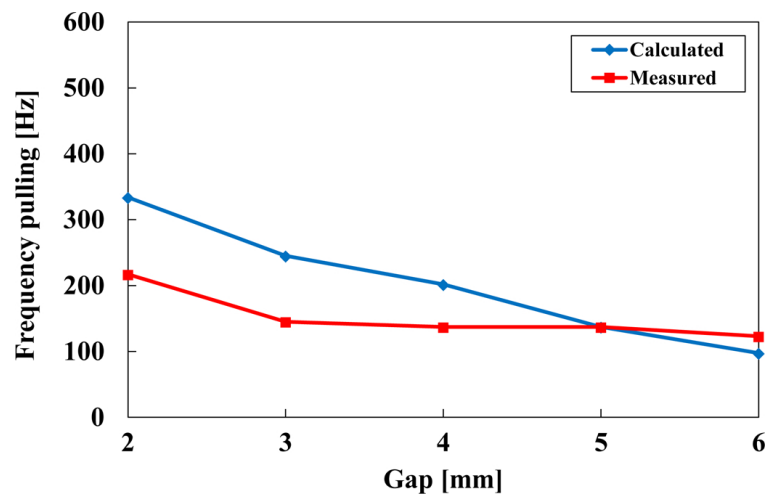

Figure 20. Calculated and measured angular frequency pulling at different gaps.

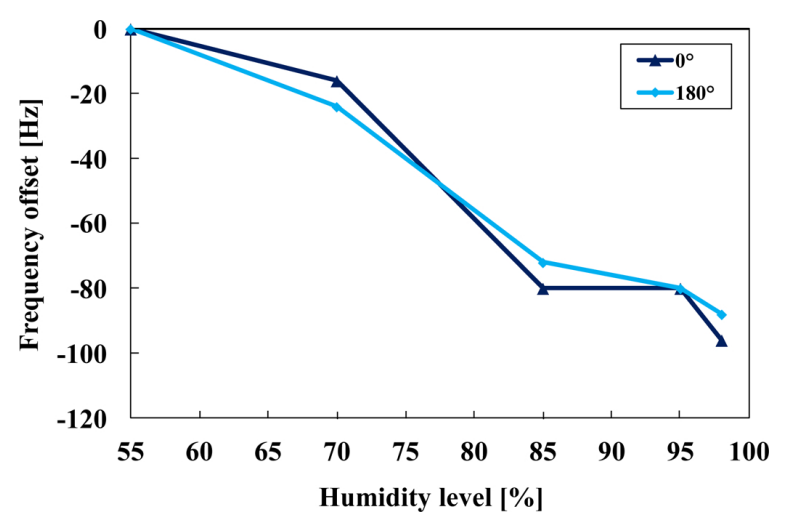

Figure 21. Measured frequency offset on a single resonator due to different humidity levels (humidity level increased every $30 \mathrm{~min}$, gap: $3 \mathrm{~mm}$ ).

\section{Conclusions}

The common approach to interrogate SAW resonators on rotating machinery is to use shorted transmission lines and to tune the element resonances to the sensor frequency in order to reach a high transmission level. However, the single resonator frequency pulling due coupler parameters variations have been reported above $2 \mathrm{kHz}(4.6 \mathrm{ppm}$ at $434 \mathrm{MHz})$ using this method. The use of ceramic antennas reduces the size of the receiver coupler element. However, the full interrogation coverage of a sensor is difficult to reach and the high $Q$ of the antenna is a source of frequency pulling. This paper presents the results obtained from a capacitive coupler based on open coplanar strip lines providing a low angular variation of transmission amplitude (less than $3 \pm 0.6 \mathrm{~dB}$ ) at different gaps. The low power detection capability of the RADARbased interrogation unit eliminates the need from the coupler elements to resonate at the sensor working frequency. This significantly reduces the parasitic pulling effect on the sensor due to the low angular variation of coupler transmission and receiver element susceptances. A $434 \mathrm{MHz}$ single resonator frequency pulling lower than $200 \mathrm{~Hz}(0.46 \mathrm{ppm})$ and $100 \mathrm{~Hz}(0.23 \mathrm{ppm})$ in a differential configuration are presented. FEM simulations show that the variation of the electrical field along the lines does not drop drastically. Moreover, frequency pulling calculations from the equivalent circuit model are presented which are in close agreement with the measurements. Furthermore, the effects of different moisture levels are also investigated which showed a frequency offset below $100 \mathrm{~Hz}$ from 55 to $98 \%$.

Acknowledgements. This work is a part of the TorqueSens project, in collaboration with SENSeOR, SANDNER Messtechnik and the Assembly and Packaging laboratory of IMTEK. This project is within the framework of the MicroTEC Südwest cluster and is funded by the German Federal Ministry of Education and Research BMBF (FKZ 16SV5192).

Edited by: B. Jakoby

Reviewed by: two anonymous referees

\section{References}

Balanis, C.: Antenna theory: analysis and design, John Wiley and Sons, 1997.

Ballandras, S., Lardat, R., Wilm, M., Pastureaud, T., Reinhardt, A., Champavert, N., Steichen, W., Daniau, W., Laude, V., Armati, R., and Martin, G.: A mixed finite element/boundary element approach to simulate complex guided elastic wave periodic transducers, J. Appl. Phys., 105, 014911, doi:10.1063/1.3021307, 2009.

Beckley, J. and Kalinin, V.: Large diameter RF rotary coupler used with a passive RF sensor, U.S. Patent No. 7782159, 2006.

Beckley, J., Kalinin, V., Lee, M., and Voliansky, K.: Non-contact torque sensors based on SAW resonators, in: Frequency Control Symposium and PDA Exhibition, 2002, IEEE International, 202-213, doi:10.1109/FREQ.2002.1075878, 2002.

Boccard, J.-M. and Reindl, L.: Printed loop and ceramic antenna for wireless interrogation of SAW resonators on a clamp system, in: Systems, Signals and Devices (SSD), 2012 9th International Multi-Conference on, 1-5, doi:10.1109/SSD.2012.6197938, 2012.

Buff, W., Rusko, M., Goroll, M., Ehrenpfordt, J., and Vandahl, T.: Universal pressure and temperature SAW sensor for wireless applications, in: IEEE Ultrasonics Symposium, 359-362, 1997.

Campbell, C.: Surface Acoustic Wave Devices and Their Signal Processing Applications, Academic Press, 1989.

DiPaolo, F.: Coplanar Strips, Networks and Devices Using Planar Transmission Lines, Boca Raton, CRC Press LLC, 2000.

Dixon, B., Kalinin, V., Beckley, J., and Lohr, R.: A Second Generation In-Car Tire Pressure Monitoring System Based on Wireless Passive SAW Sensors, in: IEEE International Frequency Control Symposium and Exposition, 374-380, 2006.

Dong, Y., Cheng, W., Wang, S., Li, Y., and Feng, G.: A multiresolution passive SAW chemical sensor, Sensor. Actuat. BChem., 76, 130-133, 2001.

El-Sa'ad, L., Darby, M., and Yates, B.: Moisture absorption by epoxy resins: The reverse thermal effect, J. Mater. Sci., 25, 35773582, doi:10.1007/BF00575392, 1990. 
Fachberger, R., Bruckner, G., Hauser, R., and Reindl, L.: Wireless SAW based high-temperature measurement systems, in: IEEE International Frequency Control Symposium and Exposition, 358-367, 2006.

Friedt, J.-M., Droit, C., Martin, G., and Ballandras, S.: A wireless interrogation system exploiting narrowband acoustic resonator for remote physical quantity measurement, Rev. Sci. Instrum., 81, 014701, doi:10.1063/1.3267311, 2010.

Friedt, J.-M., Retornaz, T., Alzuaga, S., Baron, T., Martin, G., Laroche, T., Ballandras, S., Griselin, M., and Simonnet, J.-P.: Surface acoustic wave devices as passive buried sensors, J. Appl. Phys., 109, 034905-034905-9, doi:10.1063/1.3504650, 2011.

Friedt, J.-M., Droit, C., Ballandras, S., Alzuaga, S., Martin, G., and Sandoz, P.: Remote vibration measurement: a wireless passive surface acoustic wave resonator fast probing strategy, Rev. Sci. Instrum., 83, 055001, doi:10.1063/1.4705728, 2012.

Kalinin, V.: RF Rotary Couplers for Contactless Torque Sensors Based on SAW Resonators, in: Proc. of the 22nd European Frequency and Time Forum (EFTF), Toulouse, 2008.

Kawalec, A. and Pasternak, M.: A New High-Frequency Surface Acoustic Wave Sensor for Humidity Measurement, Instrumentation and Measurement, IEEE Transactions on, 57, 2019-2023, doi:10.1109/TIM.2008.917255, 2008.

Knorr, J. and Kuchler, K.: Analysis of Coupled Slots and Coplanar Strips on Dielectric Substrate, Microwave Theory and Techniques, IEEE Transactions on, 23, 541-548, doi:10.1109/TMTT.1975.1128624, 1975.

Lecklider, T.: The world of the near field, EE-Eval. Eng., 44, p. 52, 2005.

Lonsdale, A. and Lonsdale, B.: Rotary signal coupler, U.S. Patent No. 6864759, 2001.

Ohnimus, F., Erxleben, R., Tschoban, C., Ndip, I., Niedermayer, M., Scholtz, H., Bonim, T., Guttowski, S., and Lang, K.-D.: Design and characterization of a low profile miniaturized UHF PIFA for compact wireless sensor nodes, in: Antennas and Propagation Conference (LAPC), 2010 Loughborough, 257-260, doi:10.1109/LAPC.2010.5666160, 2010.

Pohl, A., Steindl, R., and Reindl, L.: The "Intelligent Tire" utilizing passive SAW sensors - mesurement of tire friction, IEEE Transactions on Instrumentation and Measurement, 48, 1041-1046, 1999.
Reindl, L. and Shrena, I.: Wireless Measurement of Temperature Using Surface Acoustic Waves Sensors, IEEE Transactions on Utrasonics, Ferroelectrics, and Frequency Control, 51, 1457$1463,2004$.

Reindl, L., Scholl, G., Ostertag, T., Scherr, H., Wolff, U., and F. Schmidt, F.: Theory and application of passive SAW radio transponders as sensors, Ultrasonics, Ferroelectrics and Frequency Control, IEEE Transactions on, 45, 1281-1292, doi:10.1109/58.726455, 1998.

Royer, D. and Dieulesaint, E.: Elastic Waves in Solids II: Generation, Acousto-optic Interaction, Applications (Advanced Texts in Physics), Springer, 2010.

Sandacci, S. and Gilkes, J.: Rotary signal couplers, U.S. Patent Application No. 2007/0024387, 2006.

Suh, Y.-H. and Chang, K.: Coplanar stripline resonators modeling and applications to filters, Microwave Theory and Techniques, IEEE Transactions on, 50, 1289-1296, doi:10.1109/22.999141, 2002.

Tourette, S.: Etude de l'association d'elements rayonnants - capteurs SAW, Ph.D. thesis, University of Nice Sophia-Antipolis, France, 2010.

Kalinin, V., Lohr, R., Leigh, A., Beckley, J., and Bown, G.: Highspeed high dynamic range resonant SAW torque sensor for kinetic energy recovering system, in: 2010, in European Frequency and Time Forum (EFTF), doi:10.1109/EFTF.2010.6533643, 2010.

Wang, W., Lim, C., Lee, K., and Yang, S.: Wireless surface acoustic wave chemical sensor for simultaneous measurement of $\mathrm{CO}_{2}$ and humidity, J. Micro/Nanolith. MEMS MOEMS, 8, 031306, doi:10.1117/1.3158610, 2009.

White, R. and Voltmer, F.: Direct piezoelectric coupling to surface acoustic waves, Appl. Phys. Lett., 7, 314-316, 1965.

Zhang, Z.: Antenna Design for Mobile Devices, Wiley-IEEE Press, 2011. 